Gut and Liver, Vol. 11, No. 6, November 2017, pp. 843-851

\title{
Useful Endoscopic Ultrasonography Parameters and a Predictive Model for the Recurrence of Esophageal Varices and Bleeding after Variceal Ligation
}

\author{
Soung Won Jeong ${ }^{1}$, Hye Soo Kim², Sang Gyune Kim², Jeong-Ju Yoo², Jae Young Jang ${ }^{1}$, Sae Hwan Lee ${ }^{3}$, Hong Soo Kim \\ Ji Sung Lee ${ }^{4}$, Young Seok Kim², and Boo Sung Kim² \\ ${ }^{1}$ Division of Gastroenterology and Hepatology, Department of Internal Medicine, Soonchunhyang University College of Medicine, Seoul, \\ ${ }^{2}$ Digestive Disease Center and Research Institute, Department of Internal Medicine, Soonchunhyang University Bucheon Hospital, Bucheon, \\ ${ }^{3}$ Division of Gastroenterology and Hepatology, Department of Internal Medicine, Soonchunhyang University College of Medicine, Cheonan, \\ and ${ }^{4}$ Clinical Research Center, Asan Medical Center, Seoul, Korea
}

Background/Aims: To identify the usefulness of endoscopic ultrasonography with a mini-probe (EUM) and to create a predictive model for esophageal variceal (EV) recurrence and bleeding following esophageal variceal ligation (EVL). Methods: A total of 144 patients who received EUM prior to prophylactic EVL and met the inclusion criteria were enrolled. EUM findings, EV diameter, paraesophageal vein diameter, and the number of perforating veins were assessed. Results: EV recurrence was observed in 42 patients (29.2\%), 10 of whom experienced EV bleeding. Larger diameter of the paraesophageal vein (odds ratio [OR], 1.51; 95\% confidence interval [Cl], 1.17 to $1.96 ; p=0.002$ ) and perforating vein (OR, 3.27; 95\% Cl, 1.11 to 9.65; $p=0.032$ ) were significant predictive factors for EV recurrence. However, the diameter of the paraesophageal vein was the only significant risk factor for EV bleeding (adjusted OR, 1.51; 95\% Cl, 1.06 to 2.16; $p=0.022$ ). The areas under the curves of the predictive model for EV recurrence and bleeding were 0.872 (95\% Cl, 0.811 to 0.934$)$ and 0.811 (95\% $\mathrm{Cl}, 0.630$ to 0.992$)$, respectively. Conclusions: The diameter of the paraesophageal vein was a significant predictive factor for EV recurrence and bleeding. The predictive model constructed based on the significant EUM findings exhibited good performance. (Gut Liver 2017;11:843-851)

Key Words: Esophageal and gastric varices; Recurrence; Hemorrhage; Endosonography

\section{INTRODUCTION}

Portal hypertension increases blood flow and engorgement of the collateral vessels surrounding the lower esophagus and proximal stomach, leading to a build-up of the gastroesophageal varices in approximately 50\% of patients with cirrhosis. ${ }^{1}$ Once varices have been diagnosed, variceal bleeding occurs at a yearly rate of $10 \%$ to $15 \%{ }^{2}$ Esophageal variceal (EV) bleeding is associated with high morbidity and mortality in patients with cirrhosis. Treatment to prevent EV bleeding should be performed when large varices are present. Endoscopy should be considered for patients with compensated liver cirrhosis (LC) at every 2 to 3 years, and for patients with decompensated LC at every 1 to 2 years. ${ }^{3}$ Despite treatment improvements over the last decade, the overall mortality rate is $20 \%$ due to bleeding from $\mathrm{EV}^{4}$ Either nonselective $\beta$-blocker or endoscopic band ligation is recommended for the prevention of the first variceal bleeding of medium or large varices. Also, combination of $\beta$-blockers and band ligation is the preferred therapy as it results in lower rebleeding compared to either therapy alone. ${ }^{5}$ However, little is known about which patients are more prone to bleeding from EV or who need close management after EVL.

Endoscopic ultrasonography with a mini-probe (EUM) can be used to clearly visualize the vascular network around EV. The venous anatomy of the lower esophagus and upper stomach has been described in four layers, such as the intraepithelial channels, superficial venous plexus, deep venous plexus, and adventitial veins. ${ }^{6,7}$ The superficial venous plexus communicates with the deep venous plexus peri-esophageal collateral veins (peri-ECVs) and the para-esophageal collateral veins (para-ECVs)

\footnotetext{
Correspondence to: Sang Gyune Kim

Digestive Disease Center and Research Institute, Department of Internal Medicine, Soonchunhyang University Bucheon Hospital, 170 Jomaru-ro, Bucheon 14584, Korea

Tel: +82-32-621-5079, Fax: +82-32-621-6927, E-mail: mcnulty@schmc.ac.kr

Received on September 9, 2016. Revised on January 2, 2017. Accepted on January 12, 2017. Published online June 27, 2017

pISSN 1976-2283 eISSN 2005-1212 https://doi.org/10.5009/gnl16458

(a) This is an Open Access article distributed under the terms of the Creative Commons Attribution Non-Commercial License (http://creativecommons.org/licenses/by-nc/4.0) which permits unrestricted non-commercial use, distribution, and reproduction in any medium, provided the original work is properly cited.
} 
through the perforating veins. The peri-ECVs are located adjacent to the muscularis externa of the esophagus, and the paraECVs are external to the esophageal wall, with no contact with the muscularis externa. EUM enables accurate measurements of para-ECV and EV sizes and the location of the perforating veins. The collateral deep veins seen on EUM are related to recurrence of EV. ${ }^{8,9}$ However, the role of EUM for predicting EV recurrence or bleeding after EVL have not yet been established. In this study, we aimed to identify significant EUM findings to construct a predictive model for EV recurrence and bleeding followed by prophylactic EVL.

\section{MATERIALS AND METHODS}

\section{Study population}

A total of 144 of 248 patients with EV who were consecutively examined by EUM at the Digestive Disease Center and Research Institute, Department of Internal Medicine, Soonchunhyang University School of Medicine, Bucheon, Korea from December 2008 to December 2014 and met the inclusion criteria were enrolled in this study (Fig. 1). The diagnosis of liver cirrhosis was based on histological or clinical factors. The inclusion criteria were EV grade $\geq \mathrm{F} 2$ as a prerequisite and a moderate to severe red color sign, according to the general rules for recording endoscopic findings of EV, proposed by the Japanese Society for Portal Hypertension; $;^{10}$ all EUM parameters could be evaluated, including diameter of EV, diameter of paraesophageal veins, and diameter of portal veins (PVs); and baseline laboratory data available. The exclusion criteria were: failure to eradicate EV or diagnosed or developed hepatocellular carcinoma; presence of peptic ulcer or inflammatory bowel disease; concomitant main PV thrombosis; received balloonoccluded retrograde transvenous obliteration (BRTO) or transjugular intrahepatic portosystemic shunt (TIPS), EV bleeding history within 2 years prior to prophylactic EVL, death from other illness independent of liver disease, and lost to follow-up. Demographic data, including age, sex, etiology of cirrhosis, use of nonselective $\beta$-blocker, and previous episode of EV bleeding were collected. Platelet count, serum bilirubin and albumin, and prothrombin time were also examined. The severity of liver cirrhosis was graded by Child-Turcotte-Pugh (CTP) class and the Model for End-Stage Liver Disease score. Spleen size was examined by abdominal ultrasonography.

This study was approved by the Institutional Review Board (IRB) of Soonchunhyang University Bucheon Hospital (IRB number: 2015-07-022-001).

\section{Measurement of endoscopic ultrasonographic parameters}

All patients underwent EUM prior to EVL. The procedures were carried out by an endoscopist (S.G.K.) using a miniature ultrasonic probe (UM-2R, $12 \mathrm{MHz}$; Olympus, Tokyo, Japan). The endoscopy used for EUM was a GIF-2TQ 260M or a 2T240 (Olympus). The examinations were recorded on videotape so that an independent endoscopist (Y.S.K.) could review the EUM findings to avoid observer bias and discuss the images until they reached agreement. EV diameter, diameter of para-ECVs located external to the esophageal wall, diameter and number of perforating veins, and the presence of gastric varices were assessed as described previously. ${ }^{9}$ However, peri-ECVs were not evaluated entirely as many peri-ECVs occur in a single patient. The largest para-ECV that connected with EV through a perforating vein was selected as a valid parameter, but all visible

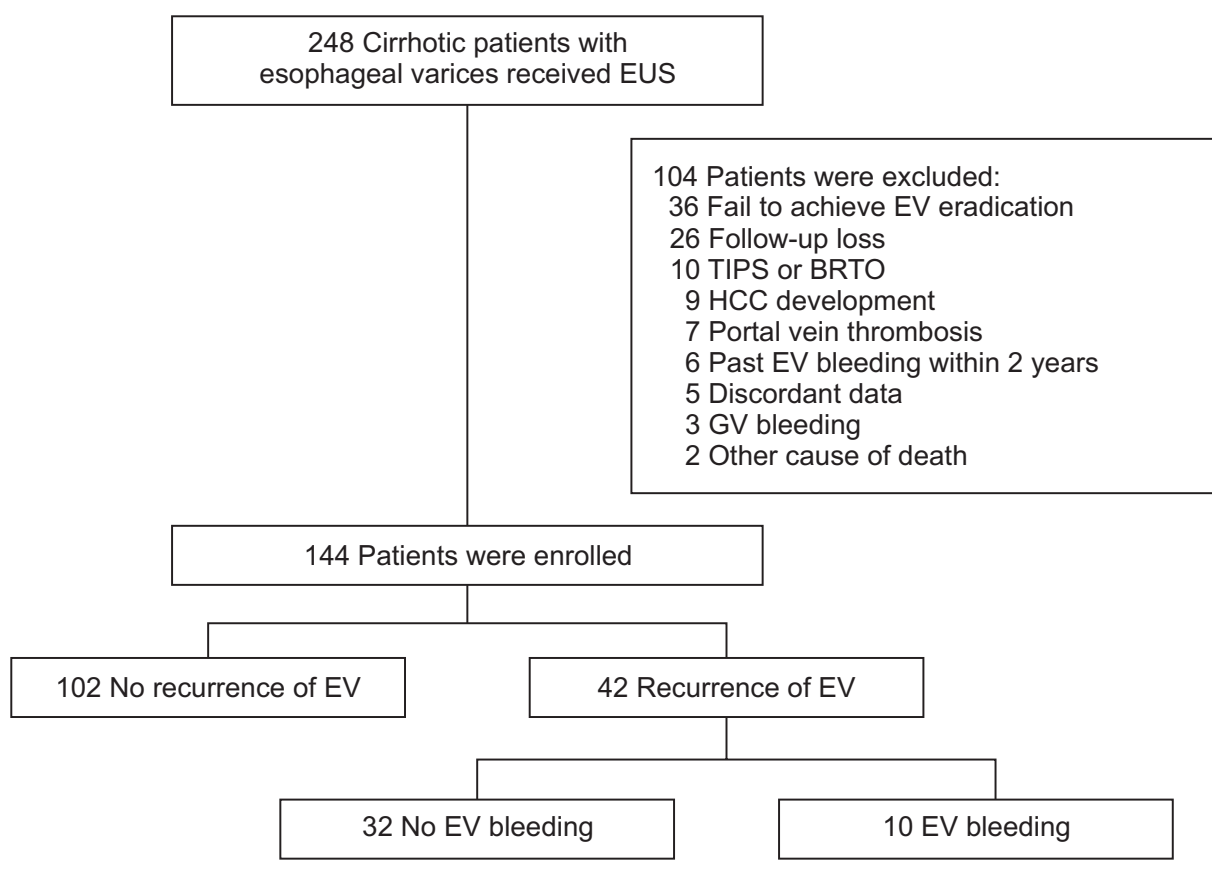

Fig. 1. Flow diagram for the study. EUS, endoscopic ultrasonography; $\mathrm{EV}$, esophageal varices; TIPS, transjugular intrahepatic portosystemic shunt; BRTO, balloon-occluded retrograde transvenous obliteration; HCC, hepatocellular carcinoma; GV, gastric varices. 


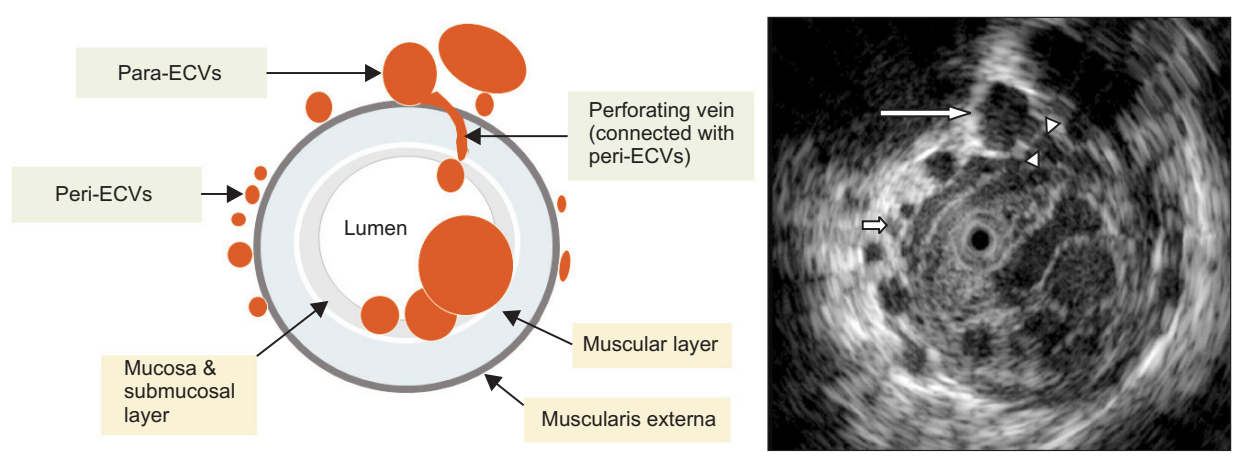

Fig. 2. A schematic diagram showing esophageal varices and vascular structures around the esophageal wall using endoscopic ultrasonography with a mini-probe. Long arrow, para-esophageal collateral veins (ECVs); short arrow, peri-ECVs; arrow head, perforating veins.

perforating veins were reported in terms of number and diameter. Esophageal varices and the vascular structures around the esophageal wall using EUM is shown as a schematic diagram in Fig. 2.

\section{EVL procedure and follow-up}

The EVL indication was based on the Baveno IV consensus. ${ }^{11}$ The endoscope loaded with a multi-banding device (6-shooter; Wilson-Cook, Limerick, Ireland) was introduced into the esophagus, and each band was consecutively applied from the gastroesophageal junction to the oral side. All visible varices were ligated during each procedure. EVL was repeated at 2-month intervals until EV were eradicated completely. The obliteration of varices was defined as nonvisualization of varices on endoscopy. Prophylactic antibiotics and omeprazole were given to all patients, and a nonselective $\beta$-blocker was prescribed to patients with $\mathrm{EV}(\geq \mathrm{F} 2)$ who had not bled but had a high risk of hemorrhage (CTP class $\mathrm{B} / \mathrm{C}$ or variceal red wale markings on endoscopy) to prevent the first variceal hemorrhage if they could tolerate a nonselective $\beta$-blocker. Follow-up endoscopy was performed at 6-month intervals for 1 year after obliteration of EV, and variceal bleeding, reappearance of EV (F1), or the presence of the red color sign was regarded as EV recurrence. Among the patients with recurred EV, bleeding was defined as any episode of hematemesis or melena from recurred EV or when endoscopy showed blood from EV.

\section{Statistical analysis}

Among the demographic and clinical parameters, continuous variables are expressed as mean and standard deviation. Differences of these variables between groups were tested with Student t-test or the Wilcoxon rank-sum test, as appropriate, after testing for normality using the Shapiro-Wilk test and for the equal variance assumption using Levene's test. Categorical variables were expressed as frequencies and percents (\%) and compared with the chi-square or Fisher exact test, as appropriate. A univariate logistic regression analysis was performed to examine the associations between the candidate predictors and the presence or absence of EV recurrence and bleeding after EVL. The odds ratio (OR) for the chance of variceal recurrence
Table 1. Patient Characteristics $(n=144)$

\begin{tabular}{|c|c|c|}
\hline \multicolumn{2}{|l|}{ Variable } & \multirow{2}{*}{$\begin{array}{c}\text { Value } \\
53.8 \pm 10.4\end{array}$} \\
\hline Age, yr & & \\
\hline \multirow[t]{2}{*}{ Sex } & Male & $114(79.2)$ \\
\hline & Female & $30(20.8)$ \\
\hline \multirow[t]{4}{*}{ Etiology } & Alcohol & $62(43.1)$ \\
\hline & Hepatitis B & $65(45.1)$ \\
\hline & Hepatitis C & $5(3.5)$ \\
\hline & Others & $12(8.3)$ \\
\hline \multirow[t]{3}{*}{ CTP class } & A & $56(38.9)$ \\
\hline & B & $67(46.5)$ \\
\hline & $\mathrm{C}$ & $21(14.6)$ \\
\hline \multirow[t]{2}{*}{ Splenomegaly } & Absence & $17(11.8)$ \\
\hline & Presence & $127(88.2)$ \\
\hline Platelet, $10^{3} / \mu \mathrm{L}$ & & $85.1 \pm 46.2$ \\
\hline \multirow[t]{2}{*}{ Previous history of EV bleeding } & Yes & $85(59.0)$ \\
\hline & No & $59(41.0)$ \\
\hline \multicolumn{3}{|l|}{ Endoscopic findings } \\
\hline \multirow[t]{2}{*}{ EV class } & $\mathrm{F} 2$ & $105(72.9)$ \\
\hline & F3 & $39(27.1)$ \\
\hline \multirow[t]{2}{*}{ Gastric varices } & Absence & $70(48.6)$ \\
\hline & Presence & $74(51.4)$ \\
\hline \multicolumn{3}{|l|}{ EUS findings } \\
\hline \multicolumn{2}{|l|}{ EV size, mm } & $8.7 \pm 2.5$ \\
\hline \multicolumn{2}{|l|}{ Para-ECV size, mm } & $7.7 \pm 2.2$ \\
\hline \multicolumn{2}{|l|}{ Perforating vein size, $\mathrm{mm}$} & $3.2 \pm 0.7$ \\
\hline \multicolumn{2}{|l|}{ Perforating vein number } & $3.5 \pm 0.7$ \\
\hline \multicolumn{2}{|l|}{ Session of EVL, $\mathrm{n}$} & $3(3-4)$ \\
\hline \multirow[t]{2}{*}{ Nonselective $\beta$-blocker } & Yes & 115 (79.9) \\
\hline & No & $29(20.1)$ \\
\hline \multirow[t]{2}{*}{ Recurrence of EV } & Absence & $102(70.8)$ \\
\hline & Presence & $42(29.2)$ \\
\hline \multirow[t]{2}{*}{ Bleeding of EV } & Absence & $134(93.1)$ \\
\hline & Presence & $10(6.9)$ \\
\hline
\end{tabular}

Data are presented as mean $\pm \mathrm{SD}$, number (\%), median (interquartile range).

CTP, Child-Turcotte-Pugh; EV, esophageal varices; EUS, endoscopic ultrasonography; ECV, esophageal collateral veins; EVL, endoscopic variceal ligation. 
and variceal bleeding was calculated for each predictor. Akaike Information Criterion (AIC)-based backward selection was used to drop insignificant variables in the multivariate logistic regression. ${ }^{12}$ The initial regression model included factors that showed the significance based on the univariate logistic regression and was further refined by the AIC-based backward selection. Multiple collinearity of continuous variables was assessed by the general variance inflation factor. ${ }^{13}$ After the final model was determined, the probability predicted for each subject was used as input to generate a receiver-operating characteristic (ROC) curve. The area under the ROC curve (AUC) and the 95\% confidence interval (CI) of this area was computed to evaluate diagnostic ability. The diagnostic test was considered to have discriminatory potential if the lower limit of the CI for the AUC was $>0.5$. The best cutoff probability value for each subject to have a recurrence of EV or bleeding after EVL was determined using Youden's index. ${ }^{14}$ Sensitivity, specificity, and positive and negative predictive values of the best cutoff value were calcu- lated. A two-tailed p-value $<0.05$ was considered significant. All statistical analyses were performed using SAS 9.4 for Windows (SAS Institute Inc., Cary, NC, USA) and R 3.1.2 version software (http://cran.r-project.org/).

\section{RESULTS}

\section{Patient characteristics}

Among the 248 patients, 144 were included in the analysis after excluding patients who failed to achieve eradication of EV ( $n=36)$, lost to follow-up ( $n=26)$, diagnosed or developed hepatocellular carcinoma $(n=9)$, concomitant main portal vein thrombosis $(n=7)$, received TIPS $(n=7)$ or BRTO $(n=3)$, EV bleeding history within 2 years prior to prophylactic EVL $(n=6)$, discordant data $(n=5)$, gastric variceal bleeding $(n=3)$, or death from another illness $(n=2)$. Males were predominant $(114 / 144$, $79.2 \%)$. Mean age was $53.8 \pm 10.4$ years. The etiologies of liver cirrhosis were hepatitis B virus $(n=65,45.1 \%)$ and alcohol $(n=62$,

Table 2. Baseline Characteristics in the Recurrence and Nonrecurrence Groups

\begin{tabular}{|c|c|c|c|c|}
\hline Variable & & $\begin{array}{l}\text { Recurrence } \\
(\mathrm{n}=42)\end{array}$ & $\begin{array}{c}\text { Nonrecurrence } \\
\quad(n=102)\end{array}$ & p-value \\
\hline Age, yr & & $53.4 \pm 9.6$ & $53.9 \pm 10.8$ & $0.790^{*}$ \\
\hline \multirow[t]{2}{*}{ Sex } & Male & $34(81.0)$ & $80(78.4)$ & $0.910^{\dagger}$ \\
\hline & Female & $8(19.0)$ & $22(21.6)$ & \\
\hline \multirow[t]{4}{*}{ Etiology } & Alcohol & $15(35.7)$ & $47(46.1)$ & $0.648^{\ddagger}$ \\
\hline & Hepatitis B & $22(52.4)$ & $43(42.2)$ & \\
\hline & Hepatitis C & $1(2.4)$ & $4(3.9)$ & \\
\hline & Others & $4(9.5)$ & $8(7.8)$ & \\
\hline \multirow[t]{3}{*}{ CTP class } & A & $7(16.7)$ & $49(48.0)$ & $<0.001^{\dagger}$ \\
\hline & B & $24(57.1)$ & $43(42.2)$ & \\
\hline & $\mathrm{C}$ & $11(26.2)$ & $10(9.8)$ & \\
\hline Splenomegaly (presence) & & $36(85.7)$ & $91(89.2)$ & 0.758 \\
\hline Platelet, $10^{3} / \mu \mathrm{L}$ & & $87.5 \pm 43.0$ & $84.1 \pm 47.6$ & $0.689^{*}$ \\
\hline Previous history of EV bleeding (yes) & & $24(57.1)$ & $61(59.8)$ & $0.913^{\dagger}$ \\
\hline \multicolumn{5}{|l|}{ Endoscopic findings } \\
\hline \multirow[t]{2}{*}{ EV class } & F2 & $22(52.4)$ & $83(81.4)$ & $<0.001^{\dagger}$ \\
\hline & F3 & $20(47.6)$ & 19 (18.6) & \\
\hline Gastric varices (presence) & & $30(71.4)$ & $44(43.1)$ & $0.004^{\dagger}$ \\
\hline \multicolumn{5}{|l|}{ EUS findings } \\
\hline EV size, $\mathrm{mm}$ & & $10.2 \pm 2.6$ & $8.1 \pm 2.2$ & $<0.001^{*}$ \\
\hline Para-ECV size, $\mathrm{mm}$ & & $9.1 \pm 2.5$ & $7.1 \pm 1.8$ & $<0.001^{*}$ \\
\hline Perforating vein size, $\mathrm{mm}$ & & $3.6 \pm 0.5$ & $3.0 \pm 0.7$ & $<0.001^{\S}$ \\
\hline Perforating vein number & & $3.6 \pm 0.9$ & $3.4 \pm 0.7$ & $0.119^{*}$ \\
\hline Session of EVL, $\mathrm{n}$ & & $3(2-4)$ & $3(2-3)$ & $0.089^{\prime \prime}$ \\
\hline Nonselective $\beta$-blocker (yes) & & $34(81.0)$ & $81(79.4)$ & $1^{\dagger}$ \\
\hline Bleeding of EV (presence) & & $10(23.8)$ & 0 & $<0.001^{\S}$ \\
\hline
\end{tabular}

Data are presented as mean \pm SD, number (\%), median (interquartile range). Variables with a p-value $<0.05$ are shown in bold. CTP, Child-Turcotte-Pugh; EV, esophageal varices; EUS, endoscopic ultrasonography; ECV, esophageal collateral veins; EVL, endoscopic variceal ligation.

${ }^{*}$ Student t-test assuming equal variance between the groups; ${ }^{\dagger}$ Chi-square test; ${ }^{\ddagger}$ Fisher exact test; ${ }^{\S}$ Student t-test assuming unequal variance between the groups; "Wilcoxon rank sum test. 
43.1\%) followed by hepatitis $C$ virus $(n=5,3.5 \%)$. The CTP classes were 56 (38.9\%) A, 67 (46.5\%) B, and 21 (14.6\%) C. Eightyfive patients (59.0\%) had a variceal bleeding history, and 115 (79.9\%) took a nonselective $\beta$-blocker. The median number of EVL sessions to achieve complete variceal eradication was three (range, 1-6). The EV classes based on the EUM findings were F2 $(\mathrm{n}=105,72.9 \%)$, and F3 ( $\mathrm{n}=39,27.1 \%)$, and gastric varices appeared in 74 patients (51.4\%). Based on the EUM findings, the mean diameters of EV, para-ECVs, and perforating veins were $8.7 \pm 2.5,7.7 \pm 2.2$, and $3.2 \pm 0.7 \mathrm{~mm}$, respectively (Table 1 ).

\section{Comparison of patients according to recurrence of EV}

During the 1 year follow-up period, EV recurred in 42 patients (29.2\%). The demographic and clinical data of the patients with (recurrence group) and without EV recurrence (nonrecurrence group) are presented in Table 2. The nonrecurrence group was mainly comprised of CTP class A ( $n=49,48.0 \%)$, whereas the majority of the recurrence group were CTP class B $(n=24$, $57.1 \%)$. The CTP class of recurrence group was significantly higher than that of the nonrecurrence group $(\mathrm{p}<0.001)$. EV class
F3 (47.6\% vs 18.6\%, p<0.001) and presence of gastric varices (71.4\% vs $43.1 \%, \mathrm{p}=0.004)$ were more commonly seen in the recurrence group than those in the nonrecurrence group. The mean diameters of EV, para-ECVs, and perforating veins in the recurrence group were significantly larger than those in the nonrecurrence group (EV, $10.2 \mathrm{~mm}$ vs $8.1 \mathrm{~mm}, \mathrm{p}<0.001$; para$\mathrm{ECV}, 9.1 \mathrm{~mm}$ vs $7.1 \mathrm{~mm}, \mathrm{p}<0.001$; perforating vein, $3.6 \mathrm{~mm}$ vs $3.0 \mathrm{~mm}, \mathrm{p}<0.001$; respectively).

\section{Multivariate analysis and the predictive model for recurrence of EV}

Several putative parameters were assessed in the 42 patients with recurrence and the 102 patients without to develop a predictive model for EV recurrence. EV class $(p<0.001)$, presence of gastric varices $(p=0.002)$, and CTP class $(p<0.001)$ were significant in the univariate logistic regression analysis. Among EUS findings, EV size $(\mathrm{p}<0.001)$, para-ECV size $(\mathrm{p}<0.001)$, and perforating vein size $(\mathrm{p}<0.001)$ were predictive of $\mathrm{EV}$ recurrence (Table 3).

Factors with $\mathrm{p}<0.05$ in the univariate analysis were entered

Table 3. Univariate and Multivariate Analyses for Predicting Esophageal Variceal Recurrence

\begin{tabular}{|c|c|c|c|c|}
\hline \multirow{2}{*}{ Variable } & \multicolumn{2}{|c|}{ Univariable } & \multicolumn{2}{|c|}{ Multivariable* } \\
\hline & OR $(95 \% \mathrm{CI})$ & p-value & OR $(95 \% \mathrm{CI})$ & $\mathrm{p}$-value \\
\hline Age, yr & $1.00(0.96-1.03)$ & 0.788 & & \\
\hline Male sex (ref: female) & $1.17(0.49-3.03)$ & 0.735 & & \\
\hline \multicolumn{5}{|l|}{ Etiology (ref: others) } \\
\hline Alcohol & $0.64(0.17-2.66)$ & 0.509 & & \\
\hline Hepatitis B & $1.02(0.29-4.18)$ & 0.973 & & \\
\hline Hepatitis C & $0.50(0.02-5.03)$ & 0.587 & & \\
\hline \multicolumn{5}{|l|}{ CTP class (ref: A) } \\
\hline $\mathrm{B}$ & $3.91(1.60-10.64)$ & 0.004 & $4.80(1.49-15.43)$ & 0.008 \\
\hline $\mathrm{C}$ & $7.70(2.47-26.01)$ & $<0.001$ & $13.06(2.65-64.27)$ & 0.002 \\
\hline Splenomegaly & $0.73(0.26-2.24)$ & 0.555 & & \\
\hline Platelet, $10^{3} / \mu \mathrm{L}$ & $1.00(0.99-1.01)$ & 0.687 & & \\
\hline Previous history of EV bleeding, $n$ & $1.12(0.53-2.31)$ & 0.768 & & \\
\hline \multicolumn{5}{|l|}{ Endoscopic findings } \\
\hline EV class F3 (ref: F2) & $3.97(1.82-8.81)$ & $<0.001$ & & \\
\hline Gastric varices & $3.30(1.55-7.38)$ & 0.002 & $2.47(0.93-6.53)$ & 0.069 \\
\hline \multicolumn{5}{|l|}{ EUS findings } \\
\hline EV size, $\mathrm{mm}$ & $1.45(1.23-1.73)$ & $<0.001$ & $1.11(0.88-1.4)$ & 0.362 \\
\hline Para-ECV size, mm & $1.61(1.31-2.03)$ & $<0.001$ & $1.51(1.17-1.96)$ & 0.002 \\
\hline Perforating vein size, mm & $7.16(3.31-17.8)$ & $<0.001$ & $3.27(1.11-9.65)$ & 0.032 \\
\hline Perforating vein number & $1.47(0.91-2.42)$ & 0.120 & & \\
\hline Session of EVL, $\mathrm{n}$ & $1.60(0.99-2.62)$ & 0.058 & & \\
\hline Nonselective $\beta$-blocker & $1.10(0.46-2.87)$ & 0.834 & & \\
\hline
\end{tabular}

p-values $<0.05$ in the univariate analysis are shown in bold.

OR, odds ratio; CI, confidence interval; CTP, Child-Turcotte-Pugh; EV, esophageal varices; EUS, endoscopic ultrasonography; ECV, esophageal collateral veins; EVL, endoscopic variceal ligation.

*The intercept of the model is -10.8856 . 
into the multivariate logistic regression analysis to determine the independent factors predicting EV recurrence. The significant EUS parameters in the multiple logistic regression were para-ECV size (OR, 1.51; 95\% CI, 1.17 to 1.96 ; $\mathrm{p}=0.002$ ), and perforating vein size (OR, 3.27; 95\% CI, 1.11 to 9.65; $p=0.032$ ). CTP class were also significant (A/B: OR, 4.80; 95\% CI, 1.49 to $15.43 ; \mathrm{p}=0.008 ; \mathrm{A} / \mathrm{C}$ : $\mathrm{OR}, 13.06 ; 95 \% \mathrm{CI}, 2.65$ to 64.27 ; $\mathrm{p}=0.002)$.

To evaluate the performance of this model for predicting EV recurrence, a ROC analysis was conducted based on the predicted probabilities derived from the final model, and sensitivity, specificity and positive and negative predictive values were determined (sensitivity, 0.71; specificity, 0.89; accuracy, 0.84 ; positive predictive value, 0.73 ; negative predictive value, 0.88; AUC, 0.872; 95\% CI of AUC, 0.811 to 0.934) (Table 4). The model with these parameters had moderate diagnostic ability for EV recurrence (Fig. 3A).

\section{Comparison of the patients according to EV bleeding}

Among the patients with EV recurrence, 10 experienced EV bleeding. The demographic and clinical parameters of patients with (bleeding group) and without EV bleeding (nonbleeding group) after EVL are presented in Table 5. Of the parameters, significant differences in para-ECV size and perforating vein size were detected by EUS. The mean diameters of para-ECVs and perforating veins in the bleeding group were significantly greater than those in the nonbleeding group (para-ECV, 11.1 $\mathrm{mm}$ vs $8.5 \mathrm{~mm}, \mathrm{p}=0.003$; perforating vein, $3.9 \mathrm{~mm}$ vs $3.5 \mathrm{~mm}$, $\mathrm{p}=0.028$; respectively).

\section{Multivariate analysis and development of the predictive model for EV bleeding}

All parameters were tested by univariate logistic regression analysis, and para-ECV size and perforating vein size were positively correlated to EV bleeding (para-ECV: OR, 1.54; 95\% CI, 1.14 to 2.23 ; $\mathrm{p}=0.010$; perforating vein: $\mathrm{OR}, 5.69 ; 95 \% \mathrm{CI}, 1.23$ to $39.96 ; p=0.044$; respectively) (Table 6).

In the multivariate logistic regression analysis using AICbased backward selection to drop insignificant terms from the model, the final model included para-ECV size $(p=0.012)$ and perforating vein size ( $\mathrm{p}=0.073$ by the likelihood ratio statistic). The adjusted OR for perforating vein size was 4.72 (95\% CI, 0.82 to $27.07 ; \mathrm{p}=0.082$ ), whereas para-ECV size was positively correlated with EV bleeding (adjusted OR, 1.51; 95\% CI, 1.06 to 2.16; $\mathrm{p}=0.022$ ) (Table 6).

To evaluate the performance of this model for predicting EV bleeding, a ROC analysis was conducted based on the predicted probabilities derived from the final model, and sensitivity, speci-

Table 4. Diagnostic Performance of the Final Model

\begin{tabular}{lccccccc}
\hline \multicolumn{1}{c}{ Model } & SEN & SPE & ACC & PPV & NPV & AUC & 95\% CI of AUC \\
\hline Nonrecurrence vs recurrence $^{*}$ & $30 / 42(0.71)$ & $91 / 102(0.89)$ & $121 / 144(0.84)$ & $30 / 41(0.73)$ & $91 / 103(0.88)$ & $0.872^{\dagger}$ & $0.811-0.934$ \\
Nonbleeding vs bleeding $^{\ddagger}$ & $8 / 10(0.8)$ & $24 / 32(0.75)$ & $32 / 42(0.76)$ & $8 / 16(0.5)$ & $24 / 26(0.92)$ & $0.811^{\S}$ & $0.630-0.992$ \\
\hline
\end{tabular}

The best cutoff values were $0.4025^{\dagger}$ and $0.2392^{\S}$ according to Youden's method.

SEN, sensitivity; SPE, specificity; ACC, accuracy; PPV, positive predictive value; NPV, negative predictive value; AUC, area under the curve; CI, confidence interval.

*The model for recurrence: $-9.18+0.45$ (gastric varices) +0.44 (para-EV [mm])+1.45 (perforating vein [mm])-1.38 (Child-Pugh class A)+0.21 (ChildPugh class C); ${ }^{\ddagger}$ The model for bleeding: $-10.96+0.41$ (para-EV [mm])+1.55 (perforating vein $[\mathrm{mm}]$ ).

A

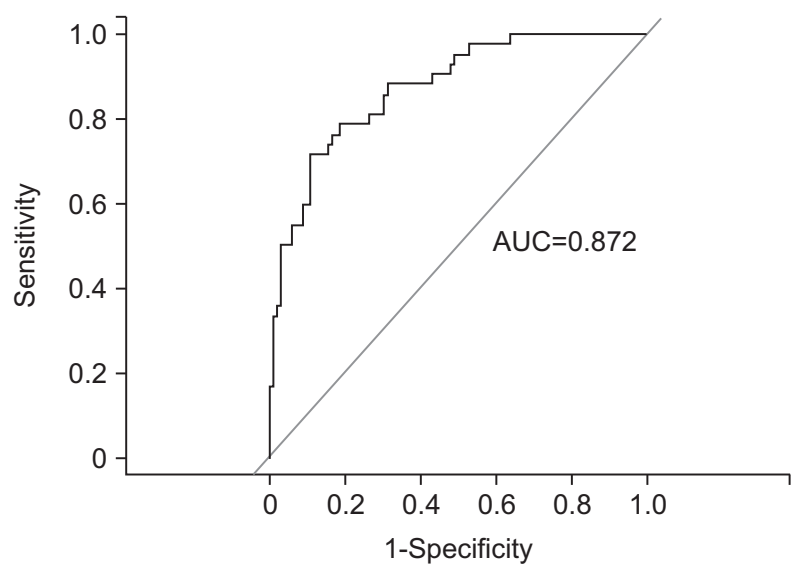

B

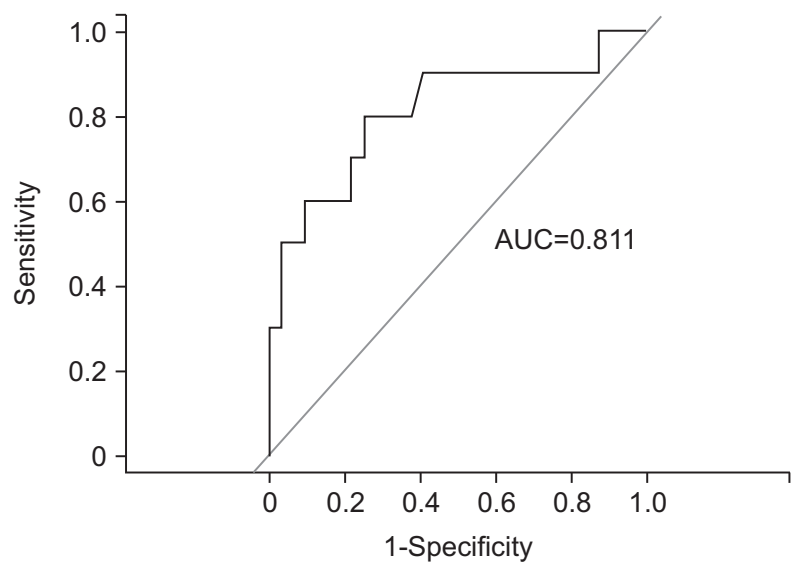

Fig. 3. Predictive model for recurrence (A) and bleeding (B) of esophageal varices after variceal obliteration. AUC, area under the curve. 
Table 5. Baseline Characteristics in the Bleeding and Nonbleeding Groups

\begin{tabular}{|c|c|c|c|c|}
\hline \multicolumn{2}{|c|}{ Variable } & \multirow{2}{*}{$\begin{array}{c}\text { Bleeding }(\mathrm{n}=10) \\
52.6 \pm 8.7\end{array}$} & \multirow{2}{*}{$\begin{array}{c}\text { Nonbleeding }(\mathrm{n}=32) \\
53.7 \pm 9.9\end{array}$} & \multirow{2}{*}{$\frac{p \text {-value }}{0.758^{*}}$} \\
\hline Age, yr & & & & \\
\hline \multirow[t]{2}{*}{ Sex } & Male & $10(100)$ & $24(75)$ & $0.165^{\dagger}$ \\
\hline & Female & 0 & $8(25)$ & \\
\hline \multirow[t]{4}{*}{ Etiology } & Alcohol & $4(40)$ & $11(34.4)$ & $0.320^{\dagger}$ \\
\hline & Hepatitis B & $5(50)$ & $17(53.1)$ & \\
\hline & Hepatitis C & $1(10)$ & 0 & \\
\hline & Others & 0 & $4(12.5)$ & \\
\hline \multirow[t]{3}{*}{ CTP class } & $\mathrm{A}$ & $1(10)$ & $6(18.8)$ & $0.689^{\dagger}$ \\
\hline & B & $7(70)$ & $17(53.1)$ & \\
\hline & $\mathrm{C}$ & $2(20)$ & $9(28.1)$ & \\
\hline \multirow[t]{2}{*}{ Splenomegaly } & Absence & 0 & $6(18.8)$ & $1^{\dagger}$ \\
\hline & Presence & $10(100)$ & 26 (81.2) & \\
\hline Platelet, $10^{3} / \mu \mathrm{L}$ & & $74.4 \pm 33.4$ & $91.6 \pm 45.3$ & $0.384^{\ddagger}$ \\
\hline \multirow[t]{2}{*}{ Previous history of EV bleeding } & Yes & $4(40)$ & $20(62.5)$ & $0.281^{\dagger}$ \\
\hline & No & $6(60)$ & $12(37.5)$ & \\
\hline \multicolumn{5}{|l|}{ Endoscopic findings } \\
\hline \multirow[t]{2}{*}{ EV class } & F2 & $5(50)$ & $17(53.1)$ & $1^{\S}$ \\
\hline & F3 & $5(50)$ & $15(46.9)$ & \\
\hline \multirow[t]{2}{*}{ Gastric varices } & Absence & $3(30)$ & $9(28.1)$ & $1^{\dagger}$ \\
\hline & Presence & $7(70)$ & 23 (71.9) & \\
\hline \multicolumn{5}{|l|}{ EUS findings } \\
\hline \multicolumn{2}{|l|}{ EV size, $\mathrm{mm}$} & $10.5 \pm 2.7$ & $10.1 \pm 2.6$ & $0.668^{*}$ \\
\hline \multicolumn{2}{|l|}{ Para-ECV size, mm } & $11.1 \pm 2.9$ & $8.5 \pm 2.0$ & $0.003^{*}$ \\
\hline \multicolumn{2}{|l|}{ Perforating vein size, $\mathrm{mm}$} & $3.9 \pm 0.4$ & $3.5 \pm 0.5$ & $0.028^{*}$ \\
\hline \multicolumn{2}{|l|}{ Perforating vein number } & $3.7 \pm 1.2$ & $3.6 \pm 0.8$ & $0.811^{*}$ \\
\hline \multicolumn{2}{|l|}{ Session of EVL, $\mathrm{n}$} & $3(2-4)$ & $3(2-4)$ & $0.851^{\ddagger}$ \\
\hline \multirow[t]{2}{*}{ Nonselective $\beta$-blocker } & Yes & $8(80)$ & $26(81.2)$ & $1^{\dagger}$ \\
\hline & No & $2(20)$ & $6(18.8)$ & \\
\hline
\end{tabular}

Data are presented as mean \pm SD, number (\%), median (interquartile range). p-values $<0.05$ are shown in bold.

CTP, Child-Turcotte-Pugh; EV, esophageal varices; EUS, endoscopic ultrasonography; ECV, esophageal collateral veins; EVL, esophageal variceal ligation.

${ }^{*}$ Student t-test assuming equal variance between the groups; ${ }^{\dagger}$ Fisher exact test; ${ }^{\ddagger}$ Wilcoxon rank sum test; ${ }^{\S}$ Chi-square test.

ficity and positive and negative predictive values were determined (sensitivity, 0.80; specificity, 0.75; accuracy, 0.76; positive predictive value, 0.50; negative predictive value, 0.92; AUC, $0.811 ; 95 \%$ CI of AUC, 0.630 to 0.992) (Table 4). The model also had moderate diagnostic ability for EV bleeding (Fig. 3B).

\section{DISCUSSION}

Esophageal varices consist of collateral vascular structures around the esophagus. ${ }^{15}$ The development of EUS and imaging systems has made it possible to observe collateral vascular structures and evaluate the vascular networks connected to variceal columns. For EV recurrence is more frequently found during EVL than in sclerotherapy, ${ }^{16,17}$ the role of EUS is more salient when doing EVL.
We investigated EUM parameters to create a predictive model for EV recurrence and bleeding after EVL. The largest diameter of para-ECVs and perforating veins, and CTP class were significant factors predicting EV recurrence, and the diameter of paraECVs was predictive of EV bleeding. The AUCs of the predictive model for EV recurrence and bleeding were 0.872 (95\% CI, 0.811 to 0.934 ), and 0.811 (95\% CI, 0.630 to 0.992 ) respectively. After all, EV recurrence happened again in nearly 30\% of our patients. These models can predict over $80 \%$ of recurrence or bleeding before initiating EVL therapy. Carvedilol or high dose nonselective $\beta$-blocker titrated with hemodynamic parameter or HVPG if available would be preferred in those who were liable to fall into recurrence or bleeding even after eradication of varices.

Several studies have evaluated the role of EUS in predicting recurrence of the EVs following EVL. Leung et al. ${ }^{9}$ and Lo et 
Table 6. Univariate and Multivariate Analyses of Candidate Predictors of Esophageal Variceal Bleeding

\begin{tabular}{|c|c|c|c|c|}
\hline \multirow{2}{*}{ Variable } & \multicolumn{2}{|c|}{ Univariable } & \multicolumn{2}{|c|}{ Multivariable $^{*}$} \\
\hline & OR $(95 \% \mathrm{Cl})$ & p-value & OR $(95 \% \mathrm{CI})$ & p-value \\
\hline Age, yr & $0.99(0.91-1.06)$ & 0.751 & & \\
\hline Platelet, $10^{3} / \mu \mathrm{L}$ & $0.99(0.97-1.01)$ & 0.276 & & \\
\hline \multicolumn{5}{|l|}{ CTP class (ref: A) } \\
\hline B & $2.47(0.33-51.17)$ & 0.439 & & \\
\hline $\mathrm{C}$ & $1.33(0.10-32.55)$ & 0.829 & & \\
\hline Previous history of EV bleeding, $\mathrm{n}$ & $2.50(0.59-11.56)$ & 0.217 & & \\
\hline \multicolumn{5}{|l|}{ Endoscopic findings } \\
\hline EV class F3 (ref: F2) & $1.13(0.27-4.83)$ & 0.863 & & \\
\hline Gastric varices & $0.91(0.20-4.95)$ & 0.909 & & \\
\hline \multicolumn{5}{|l|}{ EUS findings } \\
\hline EV size, $\mathrm{mm}$ & $1.06(0.80-1.4)$ & 0.660 & & \\
\hline Para-ECV size, mm & $1.54(1.14-2.23)$ & 0.010 & $1.51(1.06-2.16)$ & 0.022 \\
\hline Perforating vein size, $\mathrm{mm}$ & $5.69(1.23-39.96)$ & 0.044 & $4.72(0.82-27.07)$ & 0.082 \\
\hline Perforating vein number & $1.11(0.47-2.66)$ & 0.806 & & \\
\hline Session of EVL, $\mathrm{n}$ & $0.90(0.36-2.15)$ & 0.816 & & \\
\hline Nonselective $\beta$-blocker & $0.92(0.17-7.14)$ & 0.930 & & \\
\hline
\end{tabular}

p-values $<0.05$ in the analysis are shown in bold.

OR, odds ratio; CI, confidence interval; CTP, Child-Turcotte-Pugh; EV, esophageal varices; EUS, endoscopic ultrasonography; ECV, esophageal collateral veins; EVL, esophageal variceal ligation.

*The intercept of the model is -10.9586 .

al. ${ }^{18}$ reported that EV recurrence and bleeding increase significantly in patients with the severe-grade para-ECVs as observed in our study. Konishi et al. ${ }^{19}$ showed that patients experiencing EV recurrence after EVL are more likely to have severe-grade perforating veins before treatment than those without recurrence $(71.4 \%$ vs $12.5 \%, \mathrm{p}<0.01)$. Furthermore, early recurrence ( $\leq 3$ months) of EV following EVL is closely related to the presence of severe-grade cardiac perforating veins, compared to that of mild perforating veins $(90.9 \%$ vs $21 \%$, p<0.01). Masalaite et $a l .{ }^{20}$ suggested that severe peri-ECVs and multiple peri-ECVs are significant and independent prognostic factors associated with variceal recurrence risk, but not with para-ECVs or perforating veins. However, we found it very difficult to define peri-ECVs accurately because they were was too small to measure (1 to 2 $\mathrm{mm}$ ) and too numerous to count in a single patient.

Recurred EVs in our patients were newly formed beside the EVL fibrotic scars. It seemed that large diameter of para-ECVs and PVs played a role forming new collateral vessels to supply EVs after their complete obliteration. The recurrence of new EV rarely occurred when para-ECV and PV diameters were small. In addition, poor liver function as reflected by high CTP class increased the recurrence of EV. Among the patients who experienced bleeding, 84\% was Child B or C, but which was low as much as $61 \%$ in all patients. This disparity can explain why CTP class was significant as a predictor in EV recurrence, yet not in EV bleeding. If we included a larger number of patients with more advanced liver cirrhosis would be one of most important risk factors for EV bleeding as well.

The finding that para-ECV size only tended to be a significant predictor could be due to the situation where perforating veins were obliterated by band ligation, but para-ECVs were not, as they are outside of esophageal wall. Use of nonselective $\beta$-blockers and a history of EV bleeding were not associated with EV recurrence or bleeding during the follow-up period.

This study had several limitations most of which originated from the retrospective design. First, selection bias may have occurred because we did not include all the patients with significant sized EV and the study was performed in a single tertiary medical center. As a considerable number of patients failed to achieve complete eradication $(n=36)$ or were lost to follow-up $(n=26)$, the incidence of recurrence and bleeding may have been underestimated. Measurement bias may have been occurred when we determined who had complete eradication or recurrence of EV. Therefore, another expert who was blinded to the patient information reviewed and interpreted the results through videotape. Discordant results were excluded from the analysis. Since retrospectively collected data alcohol uptake that is likely to be a risk factor was not adjusted in the analysis. Third, our results can only be applied to the patients with cirrhosis who are stable and eligible to undertake EUS. However, our study included a large number of patients and followed up longer period as compared to previous studies. We hope that it can grow 
the knowledge of the hemodynamics of esophageal vascular network. Through the EUS, physicians have a more information about who and how much not to respond to EVL. Hence, they are able to decide in advance to switch to other modality or perform EVL repeatedly on a short-term basis.

Also, a future study should focus on managing patients with large para-ECVs or perforating veins to prevent EV recurrence and bleeding after EVL. The proper follow-up period and early EVL prophylaxis for these patients will help achieve complete eradication and reduce variceal bleeding.

In summary, the largest diameter para-ECVs and perforating veins were significant risk factors for EV recurrence or bleeding after EVL. The model constructed with these EUS parameters showed good predictive ability for EV recurrence and bleeding.

\section{CONFLICTS OF INTEREST}

No potential conflict of interest relevant to this article was reported.

\section{ACKNOWLEDGEMENTS}

This work was supported by the Soonchunhyang University Research Fund.

Author contribution: Conceived and designed the experiments, S.W.J., H.S.K., S.G.K. Collected the data, J.Y.J., S.H.L., H.S.K. Analyzed the data, J.S.L. Contributed to validate the data, Y.S.K., B.S.K. Wrote the paper, S.W.J., H.S.K., S.G.K., J.Y.J., S.H.L., H.S.K., J.S.L., Y.S.K., B.S.K.

\section{REFERENCES}

1. Garcia-Tsao G, Sanyal AJ, Grace ND, Carey W; Practice Guidelines Committee of the American Association for the Study of Liver Diseases; Practice Parameters Committee of the American College of Gastroenterology. Prevention and management of gastroesophageal varices and variceal hemorrhage in cirrhosis. Hepatology 2007;46:922-938.

2. North Italian Endoscopic Club for the Study and Treatment of Esophageal Varices. Prediction of the first variceal hemorrhage in patients with cirrhosis of the liver and esophageal varices. A prospective multicenter study. N Engl J Med 1988;319:983-989.

3. Suk KT, Baik SK, Yoon JH, et al. Revision and update on clinical practice guideline for liver cirrhosis. Korean J Hepatol 2012;18:121.

4. D’Amico G, De Franchis R; Cooperative Study Group. Upper digestive bleeding in cirrhosis: post-therapeutic outcome and prognostic indicators. Hepatology 2003;38:599-612.

5. de Franchis R; Baveno V Faculty. Revising consensus in portal hypertension: report of the Baveno $\mathrm{V}$ consensus workshop on methodology of diagnosis and therapy in portal hypertension. J
Hepatol 2010;53:762-768.

6. Kitano S, Terblanche J, Kahn D, Bornman PC. Venous anatomy of the lower oesophagus in portal hypertension: practical implications. Br J Surg 1986;73:525-531.

7. Hashizume M, Kitano S, Sugimachi K, Sueishi K. Three-dimensional view of the vascular structure of the lower esophagus in clinical portal hypertension. Hepatology 1988;8:1482-1487.

8. Sgouros SN, Bergele C, Avgerinos A. Endoscopic ultrasonography in the diagnosis and management of portal hypertension. Where are we next? Dig Liver Dis 2006;38:289-295.

9. Leung VK, Sung JJ, Ahuja AT, et al. Large paraesophageal varices on endosonography predict recurrence of esophageal varices and rebleeding. Gastroenterology 1997;112:1811-1816.

10. Tajiri T, Yoshida H, Obara K, et al. General rules for recording endoscopic findings of esophagogastric varices (2nd edition). Dig Endosc 2010;22:1-9.

11. de Franchis R. Evolving consensus in portal hypertension: report of the Baveno IV consensus workshop on methodology of diagnosis and therapy in portal hypertension. J Hepatol 2005;43:167176.

12. Venables WN, Ripley BD. Modern applied statistics with S. New York: Springer-Verlag, 2002.

13. Fox J, Monette G. Generalized collinearity diagnostics. J Am Stat Assoc 1992;87:178-183.

14. Youden WJ. Index for rating diagnostic tests. Cancer 1950;3:3235.

15. de Paulo GA, Ardengh JC, Nakao FS, Ferrari AP. Treatment of esophageal varices: a randomized controlled trial comparing endoscopic sclerotherapy and EUS-guided sclerotherapy of esophageal collateral veins. Gastrointest Endosc 2006;63:396-402.

16. Sarin SK, Govil A, Jain AK, et al. Prospective randomized trial of endoscopic sclerotherapy versus variceal band ligation for esophageal varices: influence on gastropathy, gastric varices and variceal recurrence. J Hepatol 1997;26:826-832.

17. Hou MC, Lin HC, Lee FY, Chang FY, Lee SD. Recurrence of esophageal varices following endoscopic treatment and its impact on rebleeding: comparison of sclerotherapy and ligation. J Hepatol 2000;32:202-208.

18. Lo GH, Lai KH, Cheng JS, Huang RL, Wang SJ, Chiang HT. Prevalence of paraesophageal varices and gastric varices in patients achieving variceal obliteration by banding ligation and by injection sclerotherapy. Gastrointest Endosc 1999;49(4 Pt 1):428-436.

19. Konishi Y, Nakamura T, Kida H, Seno H, Okazaki K, Chiba T. Catheter US probe EUS evaluation of gastric cardia and perigastric vascular structures to predict esophageal variceal recurrence. Gastrointest Endosc 2002;55:197-203.

20. Masalaite L, Valantinas J, Stanaitis J. Endoscopic ultrasound findings predict the recurrence of esophageal varices after endoscopic band ligation: a prospective cohort study. Scand J Gastroenterol 2015;50:1322-1330. 